\title{
Konzeption von Geschieberückhaltemaßnahmen unter Anwendung von 1- und 2-dimensionalen Hydrodynamik- und Geschiebetransportmodellen am Fluss Saalach
}

\author{
Designing bedload retention measures using one- and two-dimensional \\ hydrodynamics and bedload-transport models on the River Saalach \\ von S. SATTLER, P. MAYR und I. NIEDERBICHLER
}

\section{Kurzfassung/Summary}

Die gegenständliche Untersuchung umfasst die Durchführung einer 2D-Hydraulik- und Geschiebetransportmodellierung zur baulichen Optimierungunterschiedlicher Aufweitungskonzepte auf Basis einer 1-dimensionalen Geschiebeuntersuchung. Dabei werden Maßnahmen untersucht, die sowohl einen gezielten Geschieberückhalt ermöglichen, als auch eine Verbesserung der ökologischen Funktionsfähigkeit schaffen sollen. Die Aussagen bezüglich der zu erwartenden Sohlentwicklung basieren einerseits auf den Schubspannungsverteilungen und andererseits auf einer parallel durchgeführten numerischen 2DGeschiebemodellierung.

The study described in this report was intended to apply a 2D hydraulics and bedload-transport model for the structural optimisation of different channel-widening designs based on the results of a one-dimensional bedload study. The purpose was to study potential measures aiming at well-targeted bedload retention while enhancing the ecological efficiency of the river. The results relating to expected riverbed development are based on both the shearstress patterns and on numerical $2 D$ bedload modelling.

\section{Einleitung}

Aufgrund der Komplexität der Geschiebebewegung, der unterschiedlichen Transportvorgänge, sowie deren gegenseitiger Beeinflussung kann jedem Rechnen mit Geschiebemengen nur ein abschätzender Wert zukommen. Die Be- rechnung des Geschiebetriebes in Fließgewässern basiert daher in der Regel auf mehr oder weniger vereinfachten Annahmen.

Die Geschiebekornmischung in Bächen und Flüssen schwankt innerhalb weiter Grenzen und die Breiten- und Tiefenwirkung kann somit nicht allgemein und einwandfrei erfasst werden. Somit muss für jeden Gewässerabschnitt eine eigene Betrachtung der Faktoren, die den Geschiebetrieb beeinflussen, stattfinden und bei der Verwendung von Beiwerten müssen diese fallweise aus Musterstrecken des Gewässers rückschließend bestimmt werden.

Die gängigen Transportformeln, die auch in unterschiedlichen numerischen Modellen enthalten sind, eignen sich je nach Gewässertyp und Einflussfaktoren unterschiedlich gut. Daher kommt der Auswahl des Transportansatzes für die Qualität der Aussage einer Modellierung ein hoher Stellenwert zu. Die Anwendung unterschiedlicher Formeln an ein und derselben Gewässerstrecke hat diesen Umstand mehrfach aufgezeigt.

In den folgenden Ausführungen werden Ergebnisse der Anwendung unterschiedlicher Geschiebemodelle bzw. Berechnungsansätze an der Saalach vorgestellt. Die Berechnungen fanden im Rahmen von Aufträgen statt und zeigen die Anwendbarkeit für wichtige Fragestellungen in der Praxis des Flussbaus auf.

\section{Allgemeines}

Im Zuge der Untersuchung des GeschiebehaushaltsderSaalachwurde derBereichFlkm 79,00-49,00mittels eines 1D-Hydraulik- und Geschiebemodells numerisch nachgebildet und überprüft. Dieser Saalachabschnitt ist gekennzeichnet durch teils massive Anlandungsprobleme bei Hochwasser. Mittels des 1D numerischen Geschiebemodells wurde die Berechnung der gesamten Projektsstrecke durchgeführt, um Lösungsvorschläge zur Verbesserung der derzeitigen Situation zu erarbeiten und zu überprüfen.

Als Lösungskonzepte wurden u.a. Aufweitungsbereiche zur gezielten Ablagerung und periodischen Entnahme der aus dem Glemmtal eingetragenen Geschiebemengen vorgeschlagen. Die grundsätzliche Wirkung wurde mittels der 1D-Geschiebemodellierung überprüft und bestätigt.

Eine 1D-Simulation kann aber nur die prinzipielle Eignung derartiger Maßnahmen aufzeigen. Detaillierte planerische Konzeptionen erfordern eine genauere Betrachtung. Die Anordnung von Bauwerken und die resultierenden Verteilungen der Schubspannungen und Fließgeschwindigkeiten einer gewundenen Fließstrecke mit angestrebten Gleit- und Prallufereffekten erfordern zumindest eine 2D-Konzeption.

Aus diesem Grund wurde für die geplanten Aufweitungsbereiche im Saalachabschnitt Maishofen ein 2D Geschiebemodell angewandt und eine detaillierte Untersuchung bezüglich Sohlentwicklungstendenzen für unterschiedliche bauliche Varianten durchgeführt.

Ziel der Untersuchung war die Konzipierung von Maßnahmen zum gezielten Geschieberückhalt und zur Verbesserung der ökologischen Funktionsfähigkeit. 


\section{1D-Geschiebemodellierung}

\subsection{D-Modell}

Anwendung fand das Modell Mike $11 \mathrm{ST}^{\odot}$ von DHI (Danish Hydraulik Institut, http://www.dhisoftware. com/mike11). Es handelt sich um ein 1-dimensionales Hydraulik- und Geschiebetransportmodell. Das Hydrodynamikmodul löst implizite Differenzenschemata und läuft sehr stabil. Das Sedimenttransportmodul bietet verschiedene Transportformeln (Meyer-Peter Müller, Smart\&Jäggi, Engelund-Hansen ...), kann Kornverteilungen und Deckschichtbildung berücksichtigen und liefert als Ergebnis Sedimenttransport und morphologische Veränderungen.

Die Input-Parameter einer Geschiebemodellierung bestehen zum einen aus allgemeinen Input-Parametern einer Hydraulikmodellierung und speziellen Input-Parametern für die Geschiebemodellierung.

Bei den Geschiebeuntersuchungen kristallisierte sich nach einigen Versuchen und Analysen das Modell nach Smart \& Jäggi als für die Saalach am besten geeignet heraus.

Formel 1: Transportformel nach Smart \& Jäggi

$$
\phi=4\left[\left(\frac{d_{90}}{d_{30}}\right)^{0.2} I^{0.6} c \Theta^{0.5}\left(\Theta-\Theta_{c r}\right)\right]
$$

$\Phi$... Sedimenttransport

$\Theta$... Schubspannnung

c ... Widerstandsfaktor nach Chezy

I ... Gefälle

Die von Smart \& Jäggi 1983 präsentierte Transportformel basiert auf der Formel von Meyer-Peter, Müller und integriert eine Adaption des Transportbeginns (Shields-

Tab. 1: Geschieberelevante Zubringer

\begin{tabular}{lccc} 
Zubringer & Fl.km & $\begin{array}{c}\text { Geschiebe }\left[\mathbf{m}^{3}\right] \\
\text { bei HQ150 }\end{array}$ & $\begin{array}{c}\text { HQ150 } \\
{\left[\mathbf{m}^{\mathbf{3}} / \mathbf{s}\right]}\end{array}$ \\
\hline Forsthofgraben & 78,81 & 6300 & 12 \\
\hline Kammererbach & 74,89 & 14000 & 24 \\
\hline Gerlingerbach & 73,52 & 23400 & 24 \\
\hline Ruhgassingerbach & 72 & 9500 & 22 \\
\hline Urslau & 66,77 & 20000 & 245 \\
\hline Leoganger Ache & 66,77 & 20000 & 235 \\
\hline Buchweißbach & 63,48 & 101500 & 69 \\
\hline Mühlbach bei Stoissen & 62,25 & 16100 & 23 \\
\hline Dießbach & 58,6 & 10000 & 15 \\
\hline Weißbach am Hirschbichl & 53,7 & 20000 & 102 \\
\hline Schüttachgraben & 50,77 & 410000 & 103 \\
\hline
\end{tabular}

Parameter) in Abhängigkeit vom Gefälle:

Formel 2: Modifizierter ShieldsParameter

$$
\Theta_{c r}=\Theta_{0 c r} \cos \alpha\left(1-\frac{\tan \alpha}{\tan \beta}\right)
$$

$\Theta_{c r}$... modifizierter ShieldsParameter

$\Theta_{0 c r} \ldots$ Shields-Parameter

$\alpha \quad$... Azimutwinkel des Gefälles

$\beta \quad$... Scherwinkel des Sohlmaterials unter Auftrieb

Durch diese Anpassung wird der Gültigkeitsbereich erweitert:

- Sedimentdurchmesser $>0,4 \mathrm{~mm}$

- $\mathrm{D} 30 / \mathrm{D} 90<8,5$

- $0,04 \%<\mathrm{I}<20 \%$

\subsection{D-Modellierung/Kalibrie- rung}

Eine 1D-Geschiebemodellierung wurde an der Saalach zwischen Fl.km 79,0 und Fl.km 49,0 durchgeführt. Das entspricht dem Bereich zwischen der Wehranlage des KW Neumayer und dem KW ThurnSaalach. Das Sohlgefälle liegt zwischen $2 \%$ und ca. $3 \%$, im Mittel bei etwa $5 \%$.

11 Geschieberelevante Zubrin- ger wurden dabei für die Modellierung berücksichtigt (Tab. 1).

Erster Schritt der Modellierung sind der Modellaufbau und die Kalibrierung des Modells bezüglich Wasserspiegellagen. Erst das korrekt kalibrierte Modell ermöglicht eine verlässliche Geschiebemodellierung. Für das Modell mussten für alle relevanten Zubringer $\mathrm{Ab}$ flussganglinien gemäß den Werten aus einer Niederschlag-Abflussmodellierung entwickelt und entsprechend ihrer räumlichen Lage eine Übereinstimmung des zeitlichen Ablaufes erzielt werden. Durch den instationären Charakter des $\mathrm{Ab}$ flusses und die treppenartige Wiedergabe der Welle zwischen den Zubringern konnte der Durchfluss nicht für jeden Profilpunkt exakt an die vorgegebenen Werte angeglichen werden. Die Differenzen sind jedoch nur geringfügig und stellen für die folgende Geschiebemodellierung keinen Genauigkeitsverlust dar.

Basis für die Kalibrierung war die HW-Abflussganglinie an der Saalach im August 2002.

Kalibriert wurde auf die zur Verfügung gestellten Wasserspiegelwerte für die verschiedenen Abflüsse. Die Genauigkeit der Kalibrierung spiegelt sich in der Genauigkeit der gelieferten Vergleichswerte wider. 


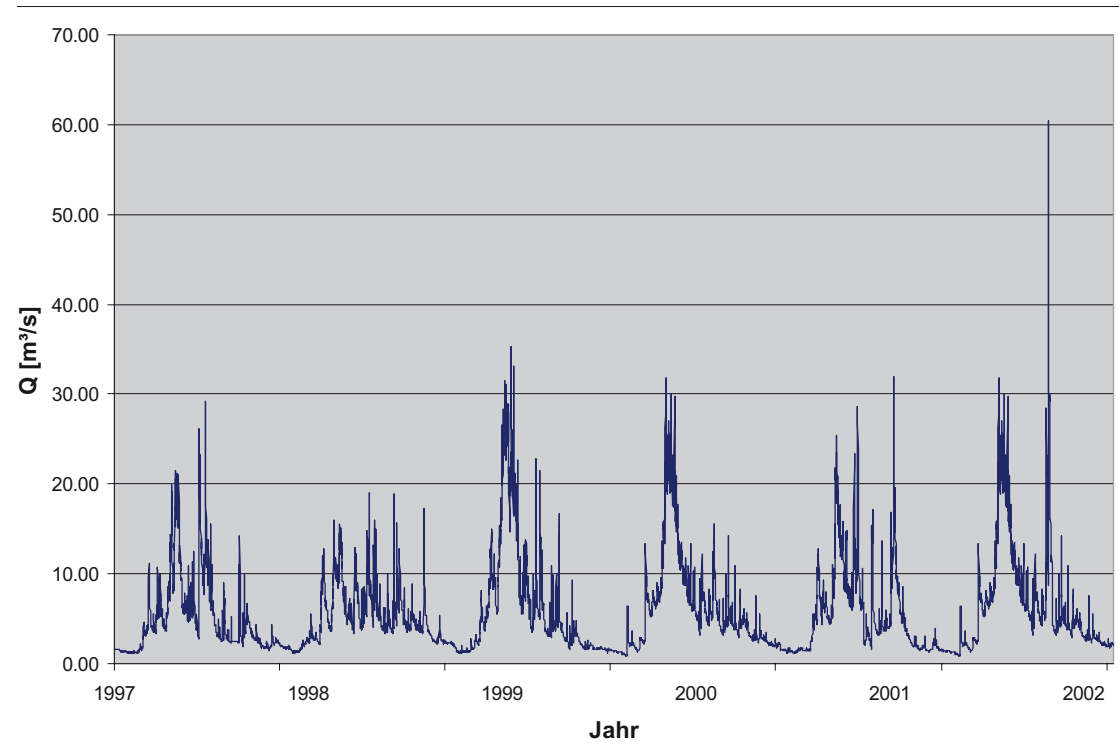

Abb. 1: 6-Jahresganglinie der Saalach bei Fl.km 79.0

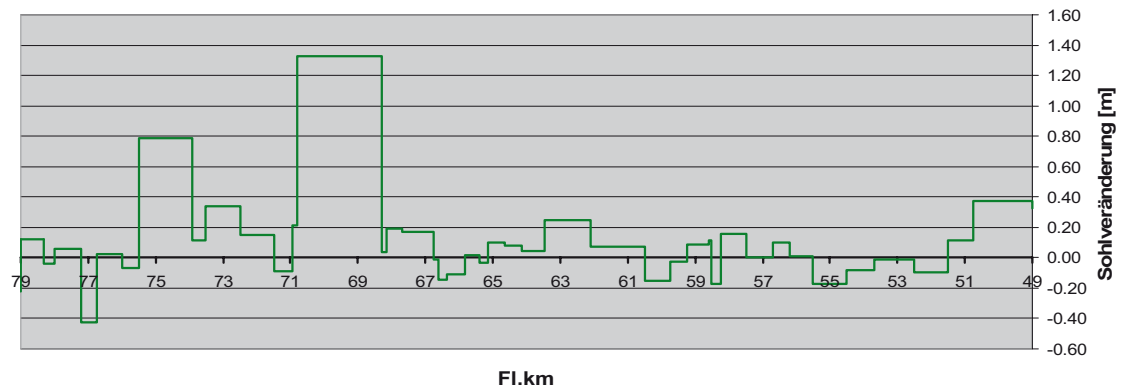

Abb. 2: Kalibrierungswerte für die Sohlveränderungen

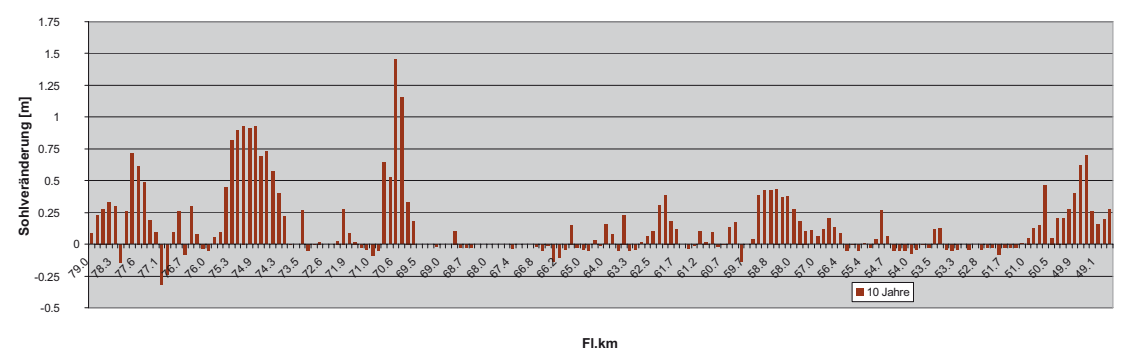

Abb. 3: Sohlveränderungen 10-Jahres-Analyse

Augrund der Tatsache, dass Geschiebetrieb erst bei höheren Wasserständen auftritt, wurde vor allem eine Übereinstimmung der Kalibrierungswerte bei $\mathrm{HW}$ angestrebt, weshalb die Kalibrierung auf HQ1 und HQ100 ausgelegt ist.

Für die Geschiebemodellierung ist es notwendig, Parameter und Randbedingungen bezüglich Geschiebe festzulegen. Neben den entsprechend der natürlichen Ausprägung folgt.

Für alle Zubringer wurde so eine mit dem Abfluss verknüpfte Geschiebeganglinie von 1997 bis 2002 (6-Jahresreihe) entwickelt. (Abb. 1)

Die Kalibrierung erfolgte anhand der gemessenen Sohlveränderungen zwischen 1997 und 2002. Dabei wurden die erfolgten Baggerungen bereichsweise eingearbeitet. (Abb 2.)

Die Schwierigkeit der Kalibrierung beruht auf der ungenauen Angabe bezüglich Geschiebeeintrag (Schätzungen), der punktuellen Erhebung von Korndurchmessern, Kornverteilungen und der Abschätzung von Deckschichtbildung etc.

Neben der modellhaften Nachbildung des hochkomplexen Vorgangs des Geschiebetransportes sind Unschärfen und Generalisierungen im Bereich der Eingangsdaten Beschränkungen für die Genauigkeit der Kalibrierung und der weiteren Ergebnisse. Die genannten Einflussfaktoren machen die Kalibrierung $\mathrm{zu}$ einem sehr zeitaufwendigen, aber notwendigen Prozess.

Grundsätzlich konnte die Sohlveränderung gut nachgebildet werden, speziell flussab der Urslaumündung (Fl.km 66,77) bis zur Mündung des Schüttachgrabens bei Fl.km 50,77. Problematischer sind die Modellränder, v. a. wegen der Wehranlagen, die das Untersuchungsgebiet oben und unten begrenzen. Auch ist der Schüttachgraben mit seinem hohen Geschiebeeintragspotential schwierig einzuschätzen und dadurch die nachfolgende Strecke kurz vor dem Abschnittsende kritisch $\mathrm{zu}$ betrachten.

\section{Szenarien und Analysen}

Mit dem kalibrierten Modell wurden Analysen von verschiedenen Szenarien durchgeführt. An erster Stelle stand eine Langzeitsimula- 


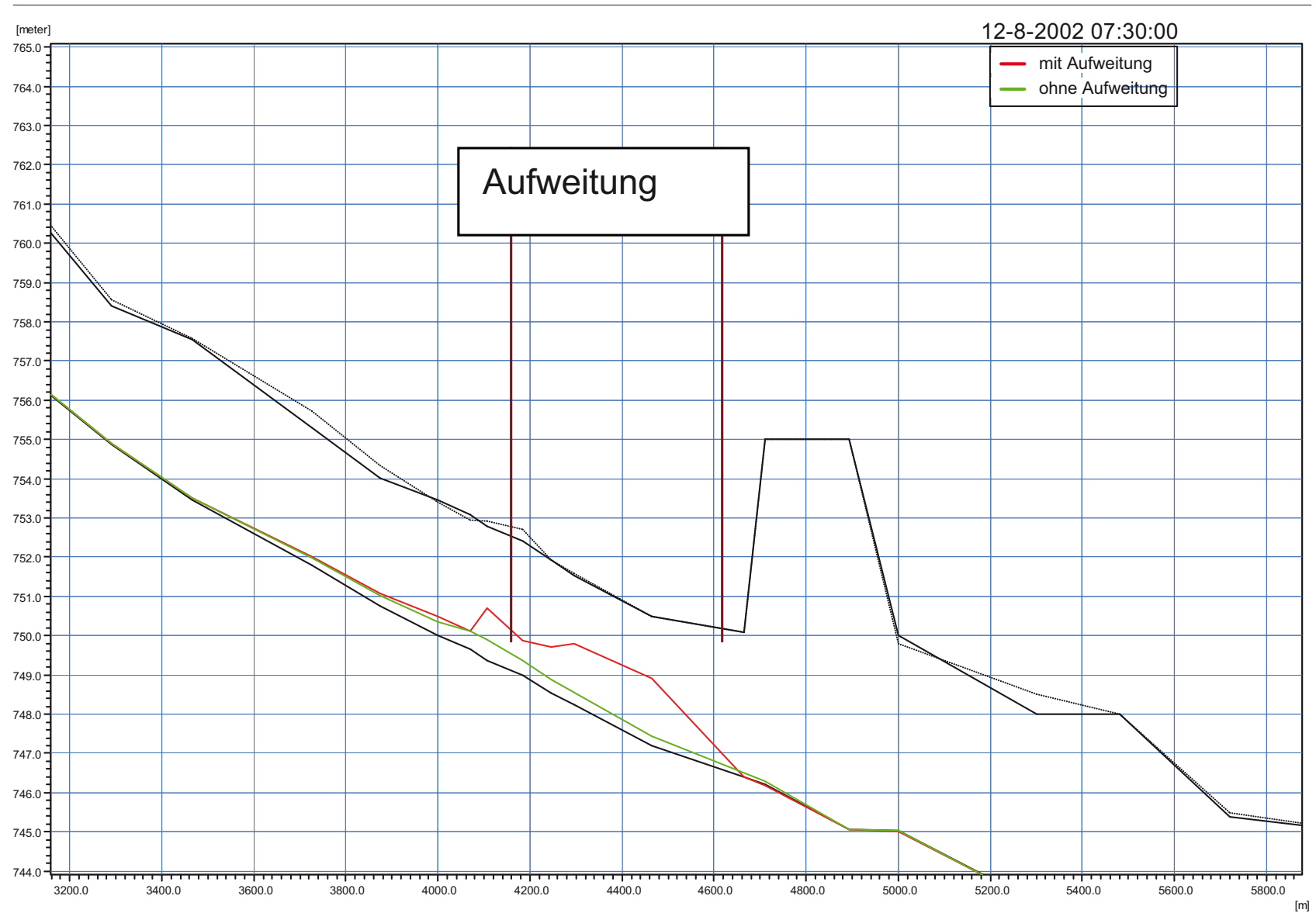

Abb. 4: Längenschnitt - Vergleich Sohlveränderung ohne (--) und mit (--) Aufweitung

tion mit unveränderter Geometrie über 10 Jahre und in Folge Untersuchungen bezüglich Aufweitungen im Bereich von Fl.km 76,0 und Fl.km 74,5.

\subsection{Langzeitsimulation}

Bei der 10-Jahres-Analyse sind deutliche Bereiche mit Anlandungstendenz und Bereiche mit Eintiefungstendenz zu erkennen. (Abb. 3) Im Zuge von Instandhaltungsmaßnahmen und Materialentnahmen nach Extremereignissen kommt es $\mathrm{zu}$ anthropogenen Sohlveränderungen, die in den Modellannahmen nicht vorgenommen wurden. Aussagen über eine Langzeitentwicklung sind dadurch nur begrenzt möglich.

\subsection{Untersuchung von Aufwei- tungen}

Zur Ermittlung der Auswirkungen von Aufweitungen wurde die Saalach im Modell zwischen Fl.km 76,0 und Fl.km 74,0 aufgeweitet. Es können in einem 1D-Modell keine Sekundärströmungen, d. h. z. B. Innen- und Außenbogeneffekte nachgebildet werden. Darum wurde auch auf genaue Ausformungen der Aufweitungen verzichtet. Die Lage der Aufweitungen richtet sich nach den vorhandenen Profilen.

\section{Variante 1}

Aufweitung der Saalach im Modell zwischen Fl.km 74,76 und Fl.km 74,34 rechtsufrig um ca. die zweifache Gerinnebreite auf einer Länge von etwa 400 m (20 fache Gerinnebreite).

Im Aufweitungsbereich kommt es $\mathrm{zu}$ Anlandungen bis $\mathrm{zu}$ über 2,0 $\mathrm{m}$ und ca. $35.000 \mathrm{~m}^{3}$ Volumen. (Abb. 4) Das Potential dürfte noch darüber liegen. Durch die massiven Anlandungen kommt es $\mathrm{zu}$ einer Reduzierung des Sohlgefälles und weitere Geschiebeeinträge in diesem Bereich führen zu Problemen bei der Nachbildung des Geschiebetransportes durch das numerische Modell.

\section{Variante 2}

Aufweitung der Saalach im Modell zwischen Fl.km 75,535 und Fl.km 75,125 linksufrig um ca. die zweifache Gerinnebreite auf einer Länge von etwa $400 \mathrm{~m}$ (20 fache Gerinnebreite).

Eine Aufweitung im Bereich von Fl.km 75,535 und Fl.km 75,125 bewirkt Anlandungen bis zu 1,7 m und es werden knapp $16.000 \mathrm{~m}^{3} \mathrm{Ge}$ schiebe zurückgehalten. (Abb. 5) 


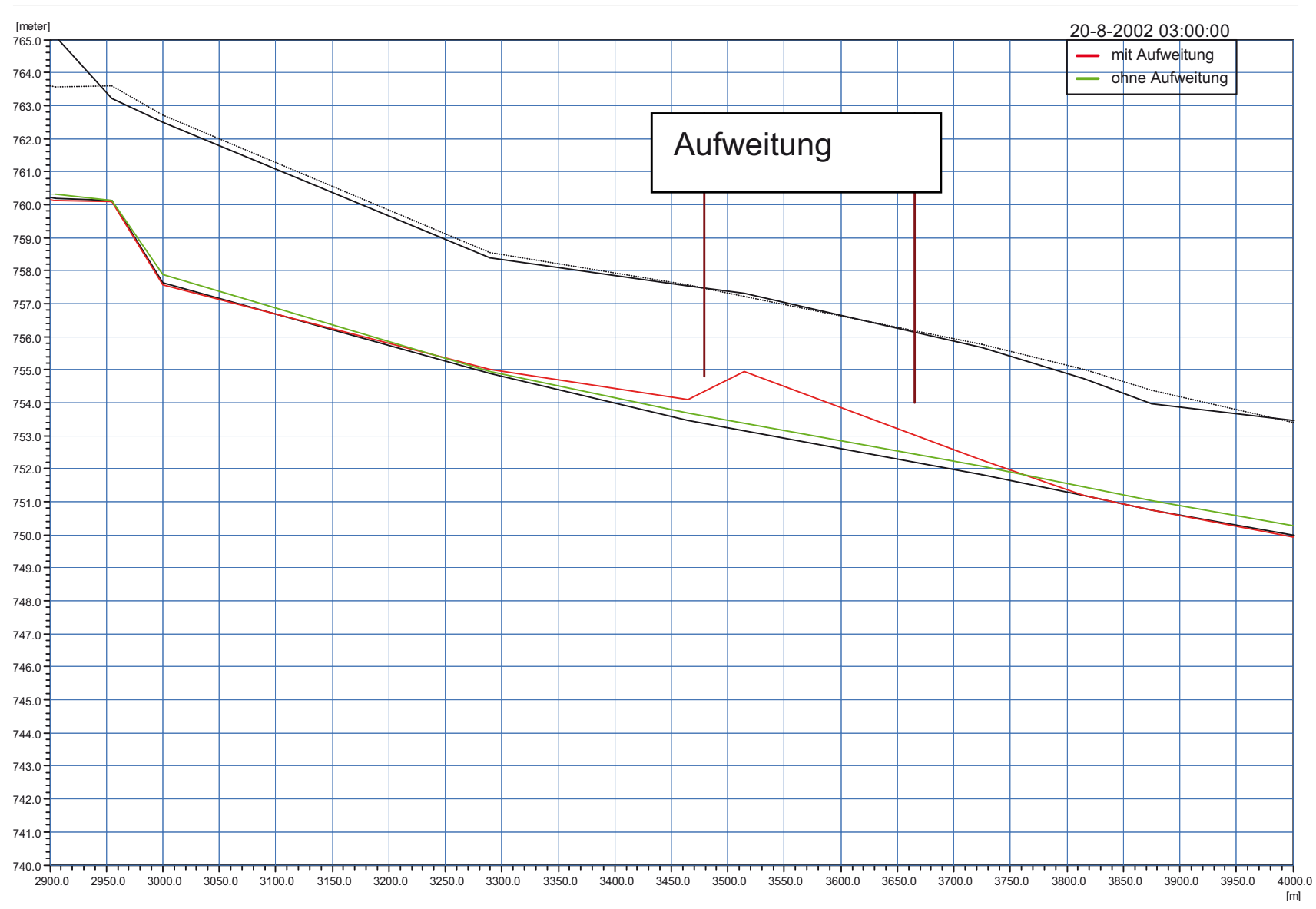

Abb. 5: Längenschnitt - Vergleich Sohlveränderung ohne (--) und mit (--) Aufweitung

Dadurch wird der folgende $\mathrm{Ab}$ schnitt entlastet.

\subsection{Variantenvergleich}

Eine Aufweitung zwischen Fl.km 75,535 und Fl.km 75,125 (Variante 2) wirkt nicht in dem Maße wie eine Aufweitung im Bereich von Fl.km 74,76 und Fl.km 74,34 (Variante 1). Grund dafür ist, dass Variante 1 erst nach der Kammererbachmündung angeordnet ist. Aus diesem Grund wäre eine Aufweitung nach diesem Zubringer zielführender. Grundsätzlich spiegeln die Ergebnisse die für die Analysen gewählte hydrologische Situation wieder, d. h. die zugrunde gelegten Abflussganglinien und der Geschiebeinput der Zubringer. Es konnte die generelle Wirksamkeit der beiden Aufweitungsvarianten nachgewiesen werden.

\subsection{Kombination der Varianten}

Zusätzlich wurde noch die Kombination beider Varianten untersucht. Dabei wurden die beiden Aufweitungen hintereinander geschalten.

Durch die Kombination beider Varianten wird die Wirksamkeit der Aufweitung zwischen Fl.km 74,76 und Fl.km 74,34 (Variante 1) reduziert, da bereits in der ersten flussauf gelegenen Aufweitung (Variante 2) ein Teil der Geschiebemenge aussedimentiert. Insgesamt werden in beiden Aufweitungen zusammen etwa $40.000 \mathrm{~m}^{3}$ Geschiebe zurückgehalten. Die flussauf gelegene Aufweitung (Variante 2) wird durch die flussab gelegene (Variante 1) kaum beeinflusst.

\section{2D-Geschiebemodellierung}

Im Anschluss an die generelle Untersuchung der Wirksamkeit von Aufweitungen hinsichtlich Geschiebetransport (1D Modell) wurden mittels einer 2D-Modellierung mehrere Aufweitungskonzepte im Detail analysiert.

Ermittelt wurde dabei die optimale Anordnung, Geometrie und Ausdehnung von Aufweitungsbereichen an der Saalach. Zusätzlich wurden bauliche Konzeptionen (Buhnen, Sohlsicherungen ...) zur Unterstützung der Anlandungstendenzen bzw. zur Sicherstellung des ungehinderten Abflusses untersucht.

Der für die Maßnahmen zur Verfügung stehende Saalachabschnitt liegt unterhalb von Maishofen zwischen Flkm 73,00 (oberhalb der Einmündung des Gerlinger- 
bachs) und Flkm 76,00 (unterhalb der Eisenbahnbrücke). Hier wurden Maßnahmenvarianten untersucht, die eine gezielte Verlandung ermöglichen sollen. Nach Hochwasserereignissen kommt es unterhalb dieses Abschnittes im Regulierungsbereich immer wieder $\mathrm{zu}$ starken Verlandungen, die zu massiven Hochwasserproblemen im Ortsbereich von Saalfelden führen.

Anhand der Ergebnisse des 2D Geschiebemodells wurde eine optimale bauliche Konzeption ermittelt, um in den Aufweitungsbereichen die aus dem Glemmtal ausgetragenen Geschiebemengen möglicht vollständig ablagern zu können.

Der Bearbeitungsaufwand einer 2D-Geschiebemodellierung ist sehr hoch. Um diesen wirtschaftlich in Grenzen zu halten, wurden für die Untersuchung Vereinfachungen für die Abflusssituation und den Geschiebeinput getroffen. Die Granulometriedaten (Sohlaufbau und Geschiebe-Input) entsprechen den beprobten Kornverteilungen. Es wurden Entwicklungstendenzen untersucht und interpretiert.

Für die Sohlentwicklungsanalyse wurde ein HQ30-Abfluss (stationär) mit einer Dauer von $16 \mathrm{~h}$ verwendet. Aufgrund des hohen Abflusswertes und der langen Abflussdauer werden die geschieberelevanten Prozesse überbetont. Dadurch kann die Entwicklungstendenz eines neuen Flussbetts beobachtet werden. Um Aussagen über die Verlandungstendenzen treffen zu können, wurde schrittweise der Geschiebeinput erhöht, bis sich Bereiche mit Verlandungstendenzen zeigten. Die Erhöhung der Geschiebefracht während eines Extremabflusses bis zur Systemkapazität erscheint durchaus geeignet die reale Verlandungssituation zu beschreiben.

Für die Berechnungen wurde das 2D-Modell $\mathrm{CCHE}^{\circ}{ }^{\odot}$ des National Center for Computational

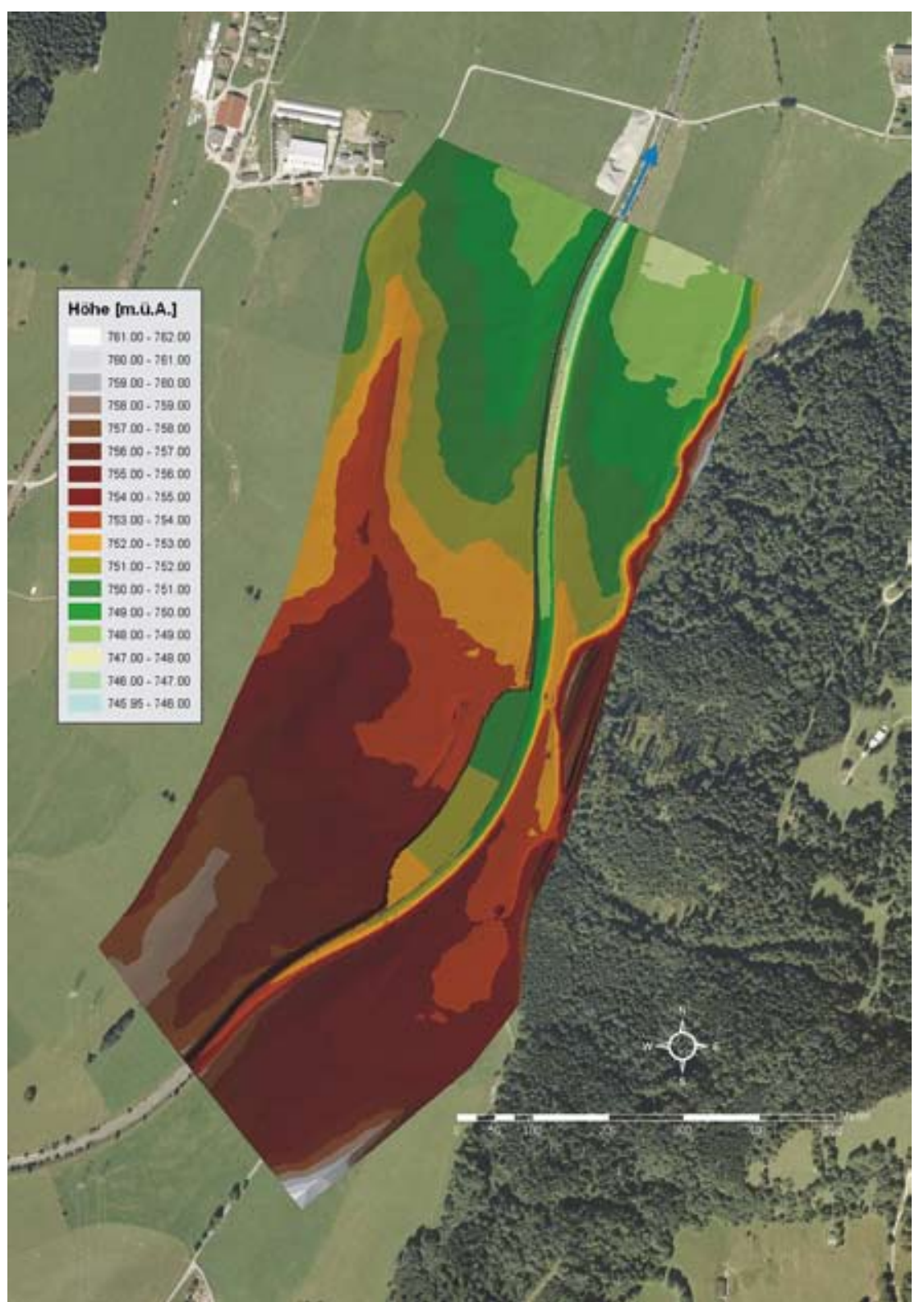

Abb. 6: Übersicht -Entwicklungsvariante (C)

Hydroscience and Engineering (NCCHE) der Universität von Mississippi (2001) verwendet.

Für eine Gesamtbetrachtung des Feststofftransports wird der gesamte Sedimenttransport (total load) im CCHE2D $^{\circ}$ Modell simuliert. Eine Unterteilung in Geschiebe (bed load) und Geschiebe plus Schwebstoff (bed load + suspended load) ist möglich. Es handelt sich beim CCHE2D-Geschiebemodul um ein Multilayer-Modell mit der Möglichkeit der Verwendung von
Korngrößenverteilungen [sowohl für Deck- und Grundschichten als auch Feststoffinput (bed load, suspended load)].

Zum Unterschied zu anderen Sedimenttransportmodellen, die ein lokales Gleichgewicht des Geschiebetransports (bed load) annehmen, ist $\mathrm{CCHE}^{2} \mathrm{D}^{\odot}$ ein vollständiges "Non-equilibrium“ Transportmodell für Geschiebe- und Schwebstofftransport (bed load + suspended load).

Für die hydraulische Kalibrie- 
rung des 2D Modells wurden die Ergebnisse der 1D-Modellierung verwendet.

\section{Modellvarianten}

Gemeinsam mit dem Auftraggeber wurden drei Basisvarianten entwickelt und untersucht.

\subsection{Variante A - Technische Konzeption}

Die technische Variante wurde auf Basis der generellen Untersuchung aufgebaut. Dabei wird im Innenbogen ein Buhnenfeld mit 8 Buhnen errichtet. Bei der Detailuntersuchung wurden die Buhnenlängen und die Gesamtlänge der Aufweitung variiert.

\subsection{Variante B - Pendelnde Konzeption}

Die Saalach entspricht im Untersuchungsbereich (bezogen auf das Gefälle und das Weiten/Tiefenverhältnis - Rosgen 1994) am ehesten einem gewundenen Flusstyp $(\mathrm{J}<2 \%$; Weite/Tiefe $>12)$. Es wurde daher als zweite Variante eine Pendelvariante untersucht.

Dabei wurde zu Untersuchungsbeginn, auf Basis der technischen Variante jeweils jede zweite linksseitige Buhne durch eine verkürzte Buhne rechtsseitig ersetzt. Die rechtsseitigen Buhnen haben eine Buhnenlänge von ca. $10 \mathrm{~m}$ und sind $80^{\circ}$-inklinant geneigt.

\subsection{Variante $\mathrm{C}$ - Entwicklungs- konzeption}

Als dritte Variante wurde eine Entwicklungsvariante definiert. Dabei wurde als Modellannahme von einem um ca. $48 \mathrm{~m}$ (Gesamtbreite etwa $60 \mathrm{~m}$ ) verbreiterten, ohne Beeinflussung durch Steuerungselemente erweiterten Aufweitungsbereich ausgegan-

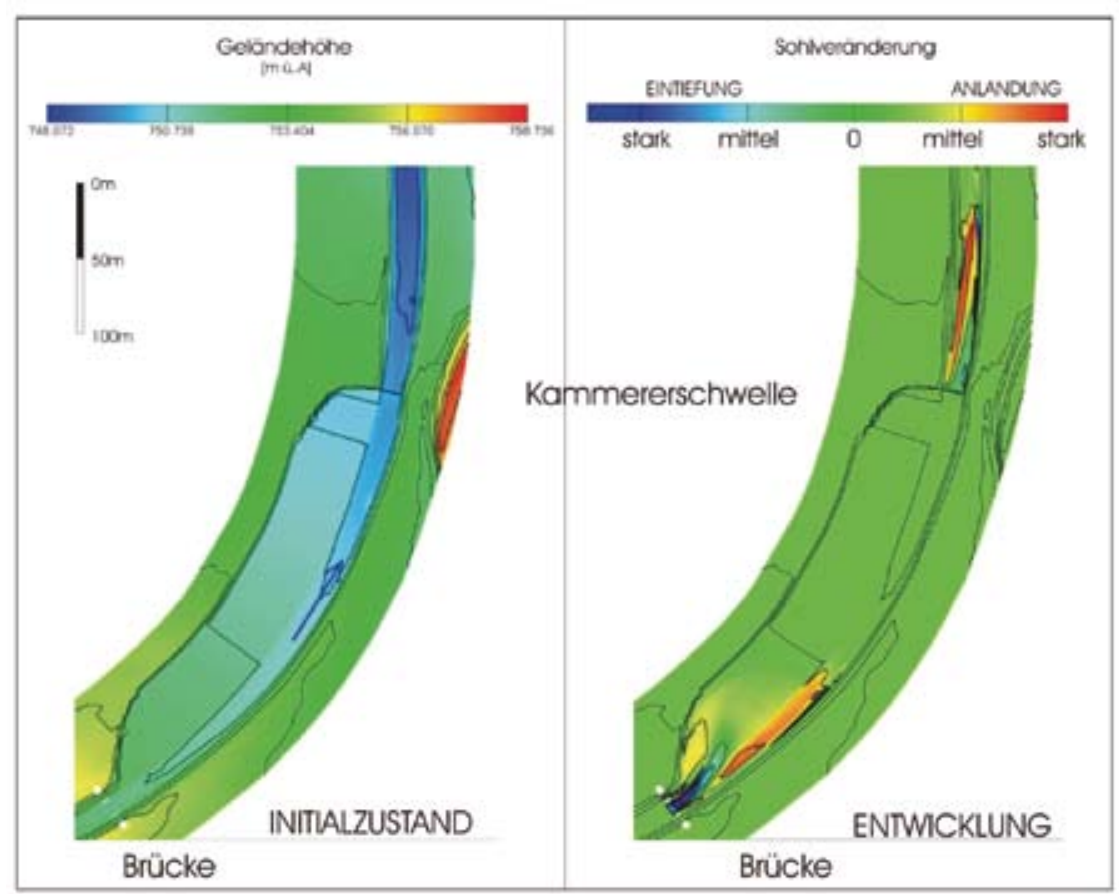

Abb. 7: Sohlveränderung - Variante C (Entwicklung)

gen. Die Entwicklung eines neuen Flusslaufs sollte dem System selbst überlassen bleiben.

Aufgrund des Umfangs der Analysen werden im Anschluss exemplarisch die Ergebnisse der Variante C - Entwicklungsvariante dargestellt. (Abb. 6)

\subsection{Bewertung}

Die Verlandungstendenzen ziehen sich bei einer unbeeinflussten Aufweitung über die gesamte Aufweitungsbreite. Die Ergebnisse der Modellannahme einer unbeeinflussten Aufweitung zeigen, dass die zusätzliche Aufweitungsbreite von etwa $40 \mathrm{~m}$ zur Bankbildung voll ausgenützt werden kann. (Abb. 7)

Aufgrund des relativ hohen Sohlgefälles $(6,5 \%$ o $)$ dürfte dieser Saalachabschnitt eher einem Umlagerungsbereich, als einem Verlandungsbereich entsprechen. Die Verlandungen resultieren aus punktuellen Einschüben aus dem Glemmtal, die die Systemkapazität oberhalb des Projektbereichs bis zur Kapazitätsgrenze ausnützen. Bis zur Kammererschwelle reduziert sich das Gefälle auf etwa 6,5\%o, unterhalb der Kammererschwelle reduziert sich das Gefälle weiter auf etwa 4,5-5\%. Der gewählte Aufweitungsbereich eignet sich als Ausschotterungsbereich, die Verlandungsneigung in den Aufweitungsbereichen zwischen Kammererschwelle und Gerlingerbach dürfte aufgrund des abnehmenden Sohlgefälles weiter zunehmen.

\subsection{Untersuchung von Steuer- elementen}

Eine vollständig freie Entwicklung birgt die Gefahr einer Sohlentwicklung über einen möglichen Maximalbereich hinaus (HochwasserGefährdung, Eigentumsrechte etc.). Um einerseits eine möglichst unbeeinflusste Sohlentwicklung $\mathrm{zu}$ ermöglichen, und andererseits einen vordefinierten Entwicklungsbereich nicht zu verlassen, wurden einige Steuerelemente und deren Auswirkung auf die Sohlentwicklung überprüft. Diese Überprüfung ist als Übergang von Entwicklungsvariante zu Pendel- 
variante (Variante B) zu sehen.

Auf Basis der Ergebnisse der Untersuchung von Variante C wurden einige zusätzliche Annahmen getroffen um die Auswirkungen einzelner Steuerelemente und deren Wirkung auf das Gesamtsystem abschätzen zu können. In Abbildung 8 sind die Ergebnisse der Zusatzuntersuchung gegenüber der ursprünglichen, unbeeinflussten Modellkonzeption dargestellt.

Fall 1: Eine Kurzbuhne (rechts) Gegenüber einer unbeeinflussten Sohlentwicklung (Initialzustand) bewirkt eine kurze rechtsufrige Buhne unterhalb der Brücke eine deutliche Verringerung der Kolkwirkung. Die Verlandungsneigung hinter der Kurzbuhne ist aber deutlich geringer als bei der unbeeinflussten Sohlentwicklung.

\section{Fall 2: Eine Langbuhne (links)}

Bei Einbau einer linksufrigen Langbuhne verlagert sich die ur- sprünglich im obersten Bereich stattfindende Sohlentwicklung flussab. Die Verlandungsneigung im linksufrig entstehenden Buhnenfeld ist deutlich geringer ausgeprägt als im unbeeinflussten Fall.

Fall 3: Drei Buhnen (zwei Kurzbuhnen rechts, eine Langbuhne links)

In Fall 3 wird eine rechtsufrige Kurzbuhne mit einer flachen linksufrigen Buhne kombiniert. Unterhalb der Langbuhne befindet sich eine weitere Kurzbuhne, um die Hauptströmrichtung nochmals in den Aufweitungsbereich zu lenken. Die linksufrige Buhne wird dabei so niedrig gehalten (Buhnenkopf auf MQ), dass sie im HQ30-Fall großteils überströmt wird. Es zeigt sich, dass die niedrige Buhne verlanden würde. Die Verlandungstendenz im obersten Aufweitungsbereich verringert sich jedoch gegenüber Fall 2.
Fall 4: Langzeitanalyse

Abschließend wurde eine Langzeitanalyse durchgeführt. Ziel war es, Erkenntnisse über gefällebzw. substrattypische Entwicklungsformen und gebietstypische Bogenradien zu erhalten. Es zeigt sich, dass die Aufweitungsbreite weitgehend ausgenützt wird. Unterhalb der Saalachbrücke wandert der rechtsufrige Anlandungsbereich Richtung flussab. Es kommt beidseitig zu deutlichen Verlandungen. Im linken Uferbereich der Aufweitung ist der Ansatz einer Gerinneverzweigung zu erkennen, die Sicherung des unbefestigten linken Uferbereichs mittels einfacher Maßnahmen (z. B. Rauhbäume) ist $\mathrm{zu}$ empfehlen. Die Bogenradien liegen zwischen ca. $120 \mathrm{~m}$ (unterhalb der Brücke) und ca. $80 \mathrm{~m}$ im Aufweitungsbereich. Die Entwicklung einer unbeeinflussten Aufweitung hängt jedoch von zahlreichen weiteren Faktoren $a b$, die in einer Langzeitanalyse erst definiert werden müssen (plötzlicher Feststoffe-

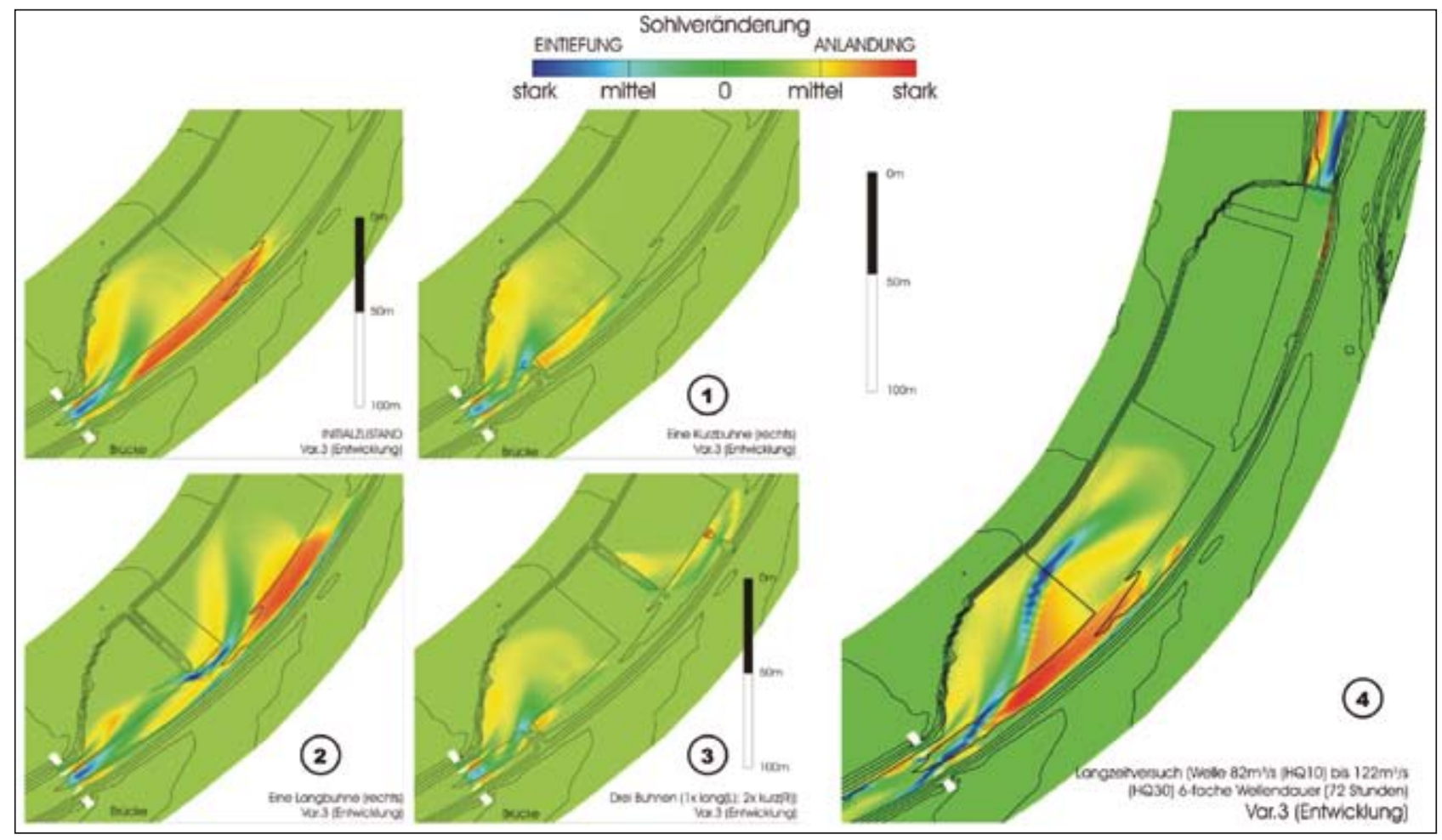

Abb. 8: Sohlveränderungen - Entwicklungskonzeptionen 
inschub, Inhomogenitäten in der Sohlsubstratzusammensetzung, Totholz, Findlinge ...).

\section{Fazit der Analysen}

Die vermutlich stärkste Verlandungstendenz zeigt eine vollständig unbeeinflusste Aufweitungsvariante. Steuerelemente sollten lediglich zur Sicherung von Bauwerken und zur Erhaltung der
Uferstabilität eingesetzt werden. Mögliche Steuerelemente sind hohe Kurzbuhnen (Buhnenköpfe auf Höhe HQ1) zur lokalen Wasserspiegelerhöhung, lange niedrige Buhnen zur Erhöhung der Verlandungsneigung und zur Strömungsumlenkung, Rauhbäume zur Ufersicherung.

Es lagen bereits umfangreiche Erfahrungen im Bereich der 1D-und 2D Feststoffmodellierung vor. Der
Aufwand der Untersuchung mittels 2D-Feststoffmodell (bewegte Sohle) überstieg den Aufwand der reinen 2D-Hydraulikmodellierung dennoch beträchtlich.

\section{Korrespondenz:}

Fa. Mayr\&Sattler OG - Ingenieurbüro für Kulturtechnik und Wasserwirtschaft

Anton Frank Gasse 13/1-2

A-1180 Wien

www.flussbau.at
DHI Water\&Environment (2004): "Mike 11 - Reference Manual"

DVWK Deutscher Verband für Wasserwirtschaft und Kulturbau (1988): Feststofftransport in Fleßgewässern - Berechnungsverfahren für die Ingenieurpraxis, Regeln der Wasserwirtschaft, H. 87/1988, Verlag Paul Parey, Bonn

Ergebnisband - Flussraumagenda Alpenraum (2006): Teil A-C; Bayerisches Staatsministerium für Umwelt, Gesundheit und Verbraucherschutz, Referat 55-Gewässer erster Ordnung; München

Habersack, H (1997): Wiener Mitteilungen. RaumZeitlichersack, Häriabiliäten im Geschiebehaushalt und dessen Beeinflussung am Beispiel der Drau. Band 144. Hrsg.: Universität für Bodenkultur, Institut für Wasserwirtschaft, Hydrologie und konstrucktiven Wasserbau.

Hunziker R P (1995): Fraktionsweiser Geschiebetransport, Mitteilungen der VAW an der ETH Zürich, Zürich

Jäggi M (1983): Die Sedimenttransportformeln von Meyer-Peter, Einstein und Englund: Vergleich , Gültigkeitsbereiche, praktische Anwendung, Arbeitsheft Nr.4 der VAW an der ETH Zürich, Zürich

Mayr, P (2002): "Hydraulische Simulationsmodelle mit Anwendungsbereich in Gebirgsbächen", Bericht im Rahmen des BMLFUW-Projekts "Gesamtheitliche Erfassung und Bewertung von Erosion- \& Transportvorgängen in Wildbacheinzugsgebieten", Institut für Alpine Naturgefahren und Forstliches Ingenieurwesen (ANFI), Universität für Bodenkultur, Wien.
Niederbichler I (2005): Kalibrierung von abiotischen Kriterien zur Entwicklung eines ökologischen Bewertungsmodells für Fliessgewässer mithilfe von mehrdimensionaler Abflussmodellierung - IST-ZUSTAND, Diplomarbeit naler Abflussmodellierung - IST-ZUSTAND, Diplomarbeit
an der Universität für Bodenkultur Wien, Institut für Wasserwirtschaft, Hydrologie und konstruktiven Wasserbau

Sattler S (1999): Numerische Simulation des Geschiebetransports an der Oberen Salzach. Wien; Diplomarbeit am Institut für Wasserwirtschaft, Hydrologie und konstruktiven Wasserbau an der Universität für Bodenkultur.

Sattler, S; Niederbichler, I; Mayr, P. Bericht AP 5 Sattler, S; Niederbichler, I; Mayr, P. Bericht AP 5 Geschiebehaushat Salach - AP5 im Rahmen des Gewässerbetreuungskonzept Saalach; Bundesministerium ür Land- und Forstwirtschaft, Umwelt und Wasserwirtschaft; Amt der Salzburger Landesregierung Fachabteilung 6/6 Wasserwirtschaft Referat 6/61Schutzwasserbau ,Gewässerpflege und kulturtechnische Maßnahmen

Sattler, S; Mayr, P: Bericht Geschieberückhaltebecken/Aufweitungsbereich Maishofen/Saalach im Rahmen des InterregllIB-Programms „Flussraumagenda im Alpenraum": Amt der Salzburger Landesregierung Fachabteilung 6/6 Wasserwirtschaft Referat 6/61Schutzwasserbau ,Gewässerpflege und kulturtechnische Maßnahmen

Schober, S (1999): Messungen des Feststoffransportes an der Oberen Drau und der Unteren Isel. Wien; Diplomarbeit am Institut für Wasserwirtschaft, Hydrologie und konstruktiven Wasserbau an der Universität für Bodenkultur.
Schoklitsch A (1934): Der Geschiebetrieb und die Geschiebefracht, Wasserkraft Wasserwirtschaft 4

Shields A (1936): Anwendung Ähnlichkeitsmechanik und der Turbulenzforschung auf die Geschiebebewegung, Mitteilung der Preussischen Versuchsanstalt für Wasserbau und Schiffbau, Berlin Heft 26

Sereinig, N (1994): Anwendung und Adaptierung eines Geschiebemodells an der Oberen Drau. Wien, Universität für Bodenkultur, Diplomarbeit.

Smart G M (1984): "Sediment transport formulae for steep channels". Journal of Hydraulic Engineering, vol.110, No.3, March 1984

Smart G M; Jaeggi, M N R (1983): "Sediment TransSmart, G M; Jaeggi, M N R (1983): "Sediment Trans-
port on Steep Slopes". Mitteilung nr. 64 of the Laboratory port on Steep Slopes". Mitteilung nr. 64 of the Laboratory
for Hydraulics, Hydrology and Glaciology at the Federal for Hydraulics, Hydrology and
Technical University, Zürich

Technical University, Zürich
Steidl, T (1991): Typologie und Abflussverhalten öserreichischer Fließgewässer - Hydrologisch-hydrographische Einteilung und regionale Gliederung, Wien.

Wasserrechtsgesetz 1959, BGBI. Nr. 215, zuletzt geandert durch das Bundesgesetz BGBI. I Nr. 156/2002 Wu, W (201): CCHE2D Sediment Transport Model Wu, W (2001). CCHE2D Sediment Transport Model ence and Engineering, Technical Report No. NCCHETR-2001-3, 2001

Zhang, Y (2006): CCHE2D - User's Manual - Version 2.210 National Center for Computational Hydroscience and Engineering, Technical Report No. NCCHETR-2006-02 